\title{
Outdoor Performance of a Thin-Film Gallium-Arsenide Photovoltaic Module
}

\author{
Timothy J Silverman*, Michael G. Deceglie*, Bill Marion*, Sam Cowley ${ }^{\dagger}$, Brendan Kayes ${ }^{\dagger}$, Sarah Kurtz \\ *National Renewable Energy Laboratory, Golden, Colorado, 80401, United States \\ ${ }^{\dagger}$ Alta Devices, Inc., Sunnyvale, California, 94085, United States
}

\begin{abstract}
We deployed a $855 \mathrm{~cm}^{2}$ thin-film, single-junction gallium arsenide (GaAs) photovoltaic (PV) module outdoors. Due to its fundamentally different cell technology compared to silicon $(\mathrm{Si})$, the module responds differently to outdoor conditions. On average during the test, the GaAs module produced more power when its temperature was higher. We show that its maximum-power temperature coefficient, while actually negative, is several times smaller in magnitude than that of a $\mathrm{Si}$ module used for comparison. The positive correlation of power with temperature in GaAs is due to temperature-correlated changes in the incident spectrum. We show that a simple correction based on precipitable water vapor (PWV) brings the photocurrent temperature coefficient into agreement with that measured by other methods and predicted by theory. The low operating temperature and small temperature coefficient of GaAs give it an energy production advantage in warm weather.
\end{abstract}

Index Terms-gallium-arsenide, thin-film, outdoor performance

\section{BACKGROUND AND INTRODUCTION}

Thin-film photovoltaic (PV) modules have typically used polycrystalline or amorphous materials deposited on inexpensive substrates. In general, the thin-film approach reduces the semiconductor material needed and can sometimes be made into a flexible, lightweight module. Single-crystal, thinfilm GaAs PV modules provide a complementary thin-film approach and have achieved higher efficiencies than their polycrystalline and amorphous counterparts [1], [2] and lowcost manufacturing processes are being developed by reusing the single-crystal substrates [3], [4]. This approach retains the advantage of minimal semiconductor material usage, providing the possibility of a high-efficiency, light-weight, thin-film PV product for a variety of applications with a cost structure that could approach that of CIGS if the process costs can be minimized.

The community has had a strong interest in integrating PV directly into roofing products, but experience has shown that PV mounted directly into a roof has elevated operating temperature, which can result in a reduction in power production on the order of $10 \%$. While it is especially problematic for direct-roof mount applications, reduced performance with heating is an issue for most $\mathrm{PV}$ products since the irradiation that drives the photovoltaic conversion also creates waste heat. A PV technology with low operating temperature, low sensitivity of power to operating temperature, or both, is highly desirable. Amorphous silicon products are known to benefit from high temperatures because of annealing that reduces the defect concentration, but amorphous silicon products have usually had efficiencies $<10 \%$. In contrast, it is expected that PV technologies that operate near the theoretical limit for efficiency should have lower operating temperature and smaller performance sensitivity to temperature.

In this work, we present for the first time an analysis of the outdoor behavior of a terrestrial, non-concentrator GaAs PV module. Throughout the work, results are illustrated by comparison with a monocrystalline Si module. First, we consider theoretical and measured variation in performance due to changes in temperature and incident spectrum. The module operates near the theoretical efficiency limit, so it is expected to have low sensitivity to temperature. After applying corrections for temperature-correlated changes in spectrum outdoors, we show that the power temperature coefficient of the GaAs module power is substantially lower than that of the $\mathrm{Si}$ module. In addition, we find that the GaAs module operates at a substantially lower temperature. We show that this difference is caused primarily by the high operating voltage and large bandgap of the GaAs module. The differences in operating temperature and in temperature coefficient lead to a difference in energy production that gives the GaAs module an advantage in hot weather. We also describe the low light performance of the GaAs module, and show that, in cloudy conditions, it performs at or above the same efficiency as in high-irradiance conditions.

\section{Method}

The GaAs module under test was fabricated by Alta Devices Inc., and is similar to a module described previously [2]. Alta Devices modules are made of thin-film single-junction GaAs solar cells. These devices are grown on reusable single-crystal GaAs substrates by metalorganic chemical vapor deposition (MOCVD), and then lifted off using the epitaxial lift-off (ELO) process [3] to create single-crystal thin-film solar cells on flexible plastic substrates. To make a module, the cells are interconnected in an overlapping "shingled" matrix to minimize shading losses. The interconnected cell matrix is then laminated to glass using standard processes.

This technology has achieved an NREL-certified worldrecord cell efficiency for single-junction devices under a nonconcentrated AM1.5G spectrum [5] of $28.8 \%$, and a worldrecord module efficiency of $24.1 \%$ under the same conditions [1]. The thin-film design allows for fundamentally higher performance than is possible for wafer-based designs, due 
to improved utilization of recycled bandedge photons. The highest efficiency that we are aware of for wafer-based singlejunction cells under a non-concentrated AM1.5 global spectrum is $26.4 \%$ [6].

The GaAs module (aperture area $855 \mathrm{~cm}^{2}$ ) and a conventional monocrystalline silicon module $\left(6,100 \mathrm{~cm}^{2}\right.$ aperture area) were deployed outdoors on the Performance and Energy Rating Testbed (PERT) at the National Renewable Energy Laboratory (NREL) Outdoor Test Facility (OTF). PERT is part of an ISO 17025 -accredited program. This facility is located at $39.7404^{\circ} \mathrm{N}, 105.1778^{\circ} \mathrm{W}$ at $1,798 \mathrm{~m}$ elevation. The modules are mounted at $40^{\circ}$ tilt, facing due south. For each of several modules, the PERT system collects a current-voltage characteristic (I-V curve), back-of-module temperature and certain meteorological measurements every fifteen minutes. Irradiance is measured using two thermopile pyranometers and a silicon photodiode. Approximately $200 \mathrm{~m}$ away at the Solar Radiation Research Laboratory (SRRL), a GPS-based instrument measures precipitable water vapor (PWV) every fifteen minutes.

Open-circuit voltage $\left(V_{\mathrm{oc}}\right)$, short-circuit current $\left(I_{\mathrm{sc}}\right)$, maximum power $\left(P_{\mathrm{mp}}\right)$ and fill factor $(F F)$ were derived from I-V curve data using ASTM E1036-08 [7]. Throughout this work, $P_{\mathrm{mp}}$ and $I_{\mathrm{sc}}$ were proportionally corrected to $1,000 \mathrm{~W} / \mathrm{m}^{2}$ irradiance using the irradiance measurement from the thermopile pyranometer. Only I-V curves meeting numerous qualityassurance criteria were considered. Among these criteria are that the irradiance measured by the fast-responding silicon photodiode pyranometer changes by less than $5 \mathrm{~W} / \mathrm{m}^{2}$ during the measurement. The data used in this work were collected from 24 July 2012 through 30 September 2012, with the exception of near-STC reference measurements collected on 17 October 2012.

Where noted, baseline performance parameters for comparing $\mathrm{GaAs}$ and $\mathrm{Si}$ performance were taken from outdoor I-V measurements on the PERT system on a cold clear day (October 17, 2012). In these cases, irradiance was between $950 \mathrm{~W} \mathrm{~m} \mathrm{~m}^{-2}$ and $1000 \mathrm{~W} \mathrm{~m}^{-2}$, and module temperature was less than $1.5^{\circ} \mathrm{C}$ from $25^{\circ} \mathrm{C}$. The performance parameters from these IV curves were corrected for incident spectrum, irradiance, and temperature.

\section{A. Effects of operating temperature on performance}

To determine the $I_{\mathrm{sc}}$ temperature coefficient that should be expected from PV cells of different types, we computed the theoretical temperature coefficients for the two materials. This was done by calculating the integral of the product of the cell's temperature-dependent quantum efficiency $(\mathrm{QE})$ with the AM1.5G reference spectrum. The temperature dependence of the QE was approximated by shifting the band-edge region of the QE curve for a $1 \mathrm{~K}$ rise above standard test conditions temperature $(298.15 \mathrm{~K})$ according to a simple approximation of band gap dependence on temperature [8], [9].

To characterize the modules' response to various ambient conditions, we first computed "apparent" temperature coefficients. A least-squares linear fit was made to $V_{\mathrm{oc}}, I_{\mathrm{sc}}, P_{\mathrm{mp}}$, or
$F F$ versus temperature using data collected when irradiance was between (950 and 1050) $\mathrm{W} / \mathrm{m}^{2}$. The slope of this fit, relative to the value of the fit at $25^{\circ} \mathrm{C}$, is the apparent temperature coefficient. This is not an invariant property of the module and is determined without accounting for changes in spectrum or angle of incidence, both of which have changes that are correlated with temperature and may have a larger effect than that of changes in temperature.

To decouple spectral effects from temperature effects, we investigated the relationship between $I_{\mathrm{sc}}$ and PWV. PWV has been shown to be a good proxy for spectrum in analysis of CdTe modules [10]. Using the same irradiance range as before, we selected data within $\pm 2.5^{\circ} \mathrm{C}$ of the median highirradiance module operating temperature. The result was a set of data taken at nearly constant irradiance and module temperature but with variable PWV. We then fitted a line to the $I_{\mathrm{sc}}$ versus PWV plot and used it to correct $I_{\mathrm{sc}}$ values to $14.2 \mathrm{~mm}$ PWV, the value used for the standard AM1.5 spectrum. The resulting set of PWV-corrected $I_{\mathrm{sc}}$ data retains a dependence on temperature that is less dependent on spectrum.

To directly measure the dependence of the I-V parameters on temperature, we performed an outdoor temperature-rise test in clear conditions at solar noon. We covered the module and allowed it to reach ambient temperature, then removed the cover to collect I-V curves as the module temperature increased. When possible, we also subsequently covered the module, allowing it to partially return to ambient temperature, periodically uncovering it briefly for additional I-V curves. The current and power measurements were then proportionally corrected for minor variations in irradiance.

\section{B. Differences in operating temperature}

To investigate differences in operating temperature, we calculated the heating power dissipated in the module from two sources: the energy from photons absorbed in the module which do not contribute to the operating point photocurrent and the excess energy of the photons that do contribute to photocurrent. These calculations are made for the Si module and the GaAs module operating under maximum power conditions for AM1.5G illumination using total spectral reflectance measurements made on a spectrophotometer with integrating sphere and measured QE. The GaAs calculation is based on an indoor I-V measurement at standard test condition (STC, $1000 \mathrm{~W} / \mathrm{m}^{2}$, AM1.5G spectrum, $25^{\circ} \mathrm{C}$ ). The $\mathrm{Si}$ calculation is based on an outdoor measurement taken on a cold clear day which was irradiance, temperature, and spectrally corrected to STC.

The power dissipated in the module from non-photocurrentproducing photons, $P_{\mathrm{abs}}$, is calculated as a function of photon energy, $E$, according to

$$
P_{\mathrm{abs}}(E)=\Phi(E)\left(1-R(E)-Q_{\mathrm{mp}}(E)\right) E,
$$

where $\Phi$ is the the photon flux, $R$ is the measured reflectance, and $Q_{\mathrm{mp}}$ is the external quantum efficiency (EQE) scaled such that the maximum power current density is given by the integral over the AM1.5G spectrum, $J_{\mathrm{mp}}=\int q Q_{\mathrm{mp}}(E) \Phi(E) d E$, 
where $q$ is the elementary charge. The excess energy of photocurrent-generating photons also contributes to heating in photovoltaic modules. While any photon with energy greater than the bandgap can produce photocurrent, only $q V_{\mathrm{mp}}$ is extracted from the module under maximum power operation. The difference between the photon energy and the energy extracted from the module is dissipated as heat from the thermalization of hot-carriers to the band edge, Joule heating, and entropic losses [11]. The power dissipated as heat in the module due to excess energy of converted photons is given by

$$
P_{\text {excess }}(E)=\Phi(E) Q_{\mathrm{mp}}(E)\left(E-q V_{\mathrm{mp}}\right) .
$$

Following the method described in [12], with the parameters suggested therein for the baseline conditions, we used calculated values for the power dissipated in each module, along with the average ambient temperature during the experiment, to calculate predicted operating temperatures for the modules.

\section{Temperature effect on energy production}

We investigated the expected difference in energy production due only to the differences in operating temperature and in temperature coefficient between $\mathrm{Si}$ and GaAs. For each technology, the module temperature rise above ambient measured during our experiment was fitted to a simple relationship,

$$
T_{\mathrm{mod}}-T_{\mathrm{amb}}=a G_{\mathrm{POA}}+T_{0 \mathrm{G}},
$$

where $T_{\bmod }$ is module temperature, $T_{\mathrm{amb}}$ is ambient temperature, $a$ is the slope of the temperature rise-irradiance line, $G_{\mathrm{POA}}$ is plane-of-array (POA) irradiance and $T_{0 \mathrm{G}}$ is the temperature rise at zero irradiance. This fit enables the prediction of the approximate module temperature using only POA irradiance and ambient temperature.

We calculated module temperature for each hour of the year using POA irradiance and ambient temperature derived from TMY2 data for Boulder, Colorado and for Phoenix, Arizona in the United States. A temperature correction factor $F_{T}$ was then calculated using

$$
F_{T}=1+\gamma\left(T_{\bmod }-25^{\circ} \mathrm{C}\right),
$$

where $\gamma$ is the temperature coefficient of $P_{\mathrm{mp}}$, independent of spectrum, calculated from the outdoor cover-uncover test. We then calculated the energy-weighted average temperature correction factor for the entire year, $\bar{F}_{T}^{y}$, which indicates the factor by which module efficiency should be multiplied to correct for the effects of operating temperature alone. If $\bar{F}_{T}^{y}$ is $<1$, operating at elevated temperature has reduced the module's energy-weighted average efficiency for the year, and thus the annual amount of energy produced, below the STC value.

\section{Irradiance effect on performance}

The irradiance dependence of the GaAs module's performance was investigated with the aid of the clearness index (CI), which is the ratio of module-plane irradiance to extraterrestrial irradiance in the same plane. This gives a metric of how clear or cloudy the sky conditions were during a particular measurement. The power measurements for this comparison were temperature- and irradiance-corrected.

\section{RESULTS AND DISCUSSION}

\section{A. Effects of operating temperature on performance}

The calculation of the theoretical temperature coefficient is illustrated in Fig. 1. The colored areas show the integrands for calculating photocurrent, which increases as the bandgap side of the QE curve shifts to lower energy with increasing temperature, due to bandgap narrowing. The theoretical temperature coefficients for short circuit current are shown on the plots and are based on the calculation at $298.15 \mathrm{~K}$ and $299.15 \mathrm{~K}$ (the curve at $400 \mathrm{~K}$ is shown for illustrative purposes). Based on this calculation the positive temperature coefficient of $I_{\mathrm{sc}}$ for GaAs is expected to be more than twice as large as for Si.

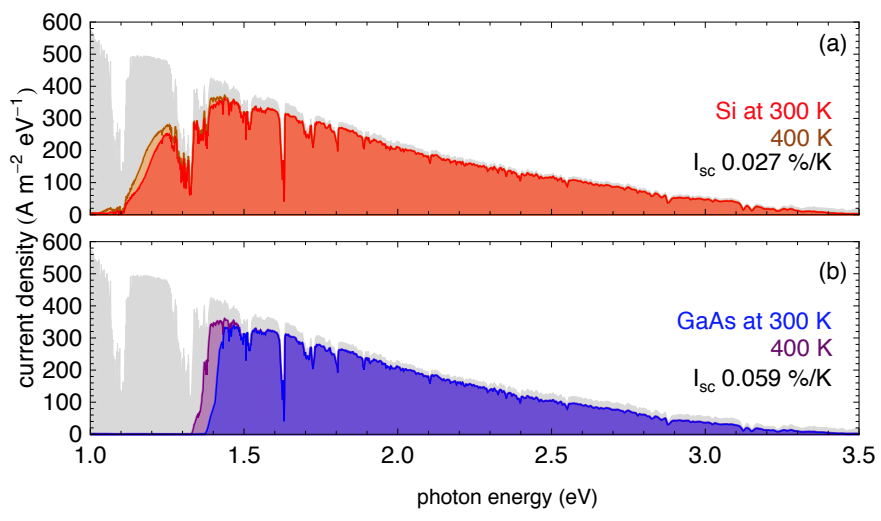

Fig. 1. Theoretical calculation of the spectral current density for Si (a) and GaAs (b) cells operating at different temperatures. The gray curve is the standard AM1.5G spectrum. Each other curve shows the product of a $\mathrm{QE}$ curve and the spectrum. This product is the integrand for calculating temperature-dependent photocurrent. The calculated values for the $I_{\mathrm{sc}}$ temperature coefficient, based on calculations at $298.15 \mathrm{~K}$ and $299.15 \mathrm{~K}$, are given in each plot.

Current has higher relative sensitivity to temperature in GaAs cells than in Si cells for two reasons. First, the bandgap of GaAs shifts more with increasing temperature than the bandgap of $\mathrm{Si}$. Second, the larger bandgap of GaAs cells means less of the solar spectrum can be collected in the first place. It is also apparent from Fig. 1 that the two technologies respond differently to changes in spectrum. In particular, the Si QE overlaps with more of the near-infrared water absorption bands than the GaAs QE.

The $P_{\mathrm{mp}}$ of the two modules, measured outdoors and corrected to $1,000 \mathrm{~W} / \mathrm{m}^{2}$, is plotted against temperature in Fig.2a. In this work, plots of the I-V curve parameters are normalized to the value of the linear fit at $25^{\circ} \mathrm{C}$. The slope of the GaAs fit is unexpectedly positive. Note also that although the data were collected on the two modules at the same time and under the same ambient conditions, the module temperature of the $\mathrm{GaAs}$ module is noticeably lower than that of the Si module. The dependence of $V_{\mathrm{oc}}$ on temperature is illustrated in Fig. 3 
The loss of $V_{\text {oc }}$ for a given increase in temperature is more than twice as large in the Si module.

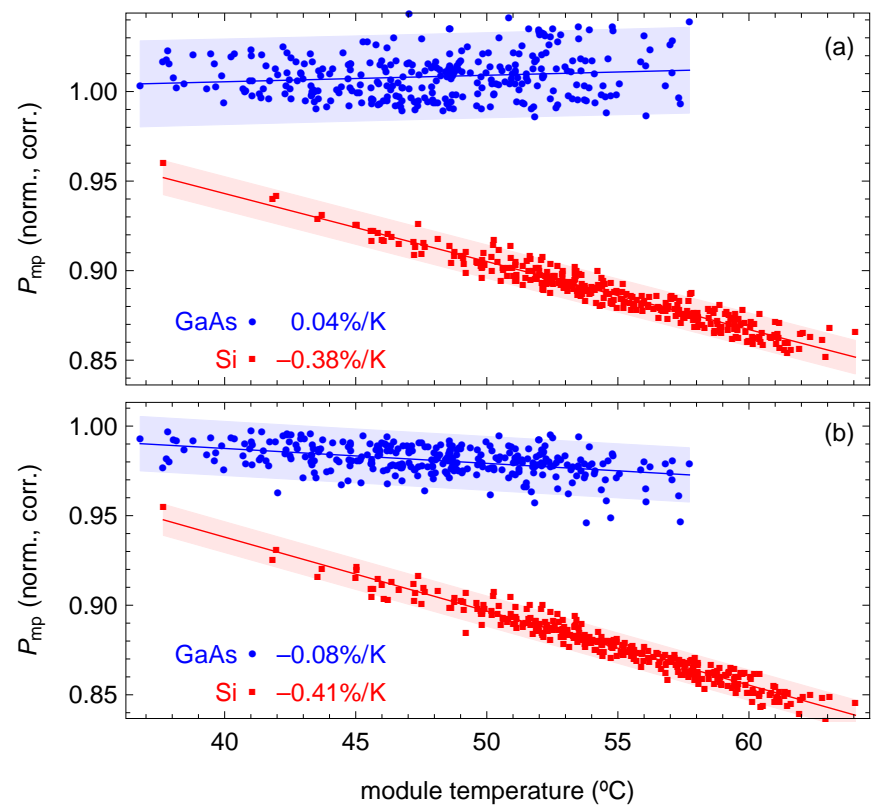

Fig. 2. $\quad P_{\mathrm{mp}}$, measured between $(950$ and 1050$) \mathrm{W} / \mathrm{m}^{2}$, normalized to $P_{\mathrm{mp}, 0}$ and corrected to $1,000 \mathrm{~W} / \mathrm{m}^{2}$ is plotted against temperature. The data in (b) are corrected to PWV of $14.2 \mathrm{~mm}$ using the relationship in Fig. 4 The linear fits in (b) provide a spectrum-independent $P_{\mathrm{mp}}$ temperature coefficient.

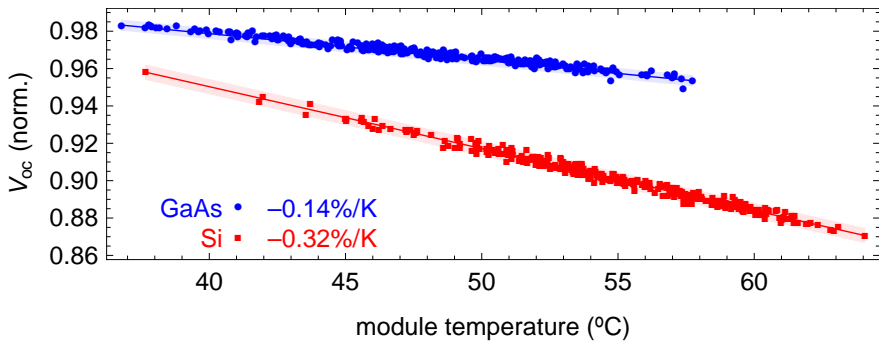

Fig. 3. $V_{\mathrm{oc}}$, filtered between (950 and 1050) $\mathrm{W} / \mathrm{m}^{2}$ and normalized to $V_{\mathrm{oc}, 0}$ is plotted against temperature. Linear fits, single-prediction $95 \%$ confidence bands and slopes relative to $V_{\mathrm{oc}}$ at $25^{\circ} \mathrm{C}$ are shown.

The positive $P_{\mathrm{mp}}$ trend in GaAs is due to the positive $I_{\mathrm{sc}}$ temperature dependence exceeding the small, negative $V_{\mathrm{oc}}$ temperature dependence. The $F F$ of the GaAs module is nearly independent of temperature.

We propose that the apparent sensitivity of $I_{\mathrm{sc}}$ to temperature is due to its dependence on spectral variables that are correlated with temperature. PWV was chosen as a simple scalar to represent these spectral changes. The fit shown in Fig. 4 was used to correct $I_{\mathrm{sc}}$ and $P_{\mathrm{mp}}$ for spectral influence.

$I_{\mathrm{sc}}$ increases with increasing PWV because water absorbs mainly portions of the solar spectrum that are unavailable to the solar cell. So an increase in PWV causes a moderate decrease in total irradiance but only a small decrease in the energy available to the cell. This causes the positive slope in irradiance-corrected $I_{\mathrm{sc}}$ with PWV. Fig. 11 shows that the small decrease in photocurrent due to the water-absorption bands is expected to be even smaller in $\mathrm{GaAs}$ than in $\mathrm{Si}$, explaining the order of the slopes in Fig. 4

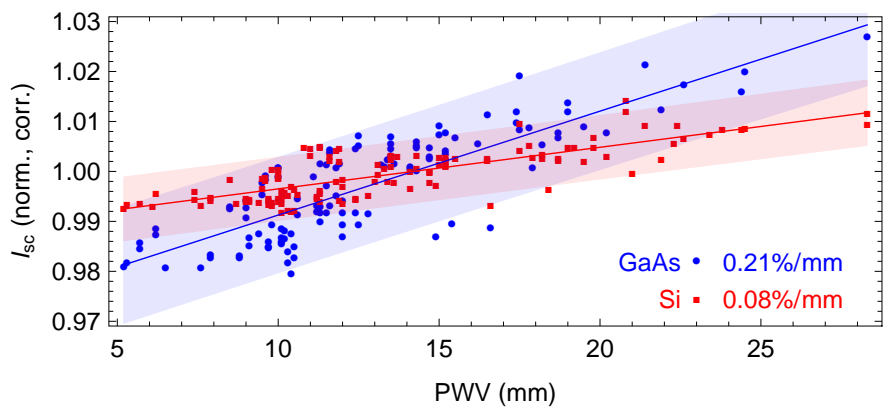

Fig. 4. $I_{\mathrm{sc}}$ collected with irradiance between $(950$ and 1050$) \mathrm{W} / \mathrm{m}^{2}$, module temperature within $\pm 2.5^{\circ} \mathrm{C}$ of median high-irradiance operating temperature, corrected to $1000 \mathrm{~W} / \mathrm{m}^{2}$ and normalized to its value at $14.2 \mathrm{~mm} \mathrm{PWV}$ is plotted against PWV. Linear fits, single-prediction 95\% confidence bands and slopes relative to $I_{\mathrm{sc}}$ values at $14.2 \mathrm{~mm} \mathrm{PWV}$ are shown.

By applying the $I_{\mathrm{sc}}$ PWV correction to $P_{\mathrm{mp}}$ we found the temperature coefficients to be $-0.08 \% / \mathrm{K}$ for $\mathrm{GaAs}$ and $-0.41 \% / \mathrm{K}$ for $\mathrm{Si}$, as shown in Fig. 2 p. Thus the $P_{\mathrm{mp}}$ temperature coefficient for GaAs is not actually positive, but it is substantially smaller than for Si. Because of its relative insensitivity to temperature, temperature-correlated spectral effects caused the GaAs module to perform better in conditions where it was also operating at higher temperature.

Each outdoor temperature-rise test resulted in multiple I-V curves collected at irradiance near $1000 \mathrm{~W} / \mathrm{m}^{2}$ and covering temperature ranges of $\geq 9 \mathrm{~K}$. After correcting to $1,000 \mathrm{~W} / \mathrm{m}^{2}$, the $I_{\mathrm{sc}}$ and $P_{\mathrm{mp}}$ temperature coefficients were determined and are compared to the temperature coefficients determined by the other methods in Table I It appears that the PWV correction eliminates most, but not all, of the spectral effect on the temperature coefficients.

TABLE I

$I_{\mathrm{sc}}$ and $P_{\mathrm{mp}}$ temperature coefficients determined using three methods.

\begin{tabular}{r|lc|ll} 
& \multicolumn{2}{|c|}{$\mathrm{GaAs}$} & \multicolumn{2}{c}{$\mathrm{Si}$} \\
method & $I_{\mathrm{sc}}(\% / \mathrm{K})$ & $P_{\mathrm{mp}}(\% / \mathrm{K})$ & $I_{\mathrm{sc}}(\% / \mathrm{K})$ & $P_{\mathrm{mp}}(\% / \mathrm{K})$ \\
\hline apparent (uncorr.) & $0.20 \pm 0.03$ & $0.04 \pm 0.03$ & $0.10 \pm 0.01$ & $-0.38 \pm 0.01$ \\
PWV correction & $0.07 \pm 0.04$ & $-0.08 \pm 0.05$ & $0.05 \pm 0.03$ & $-0.41 \pm 0.05$ \\
uncover test & $0.08 \pm 0.02$ & $-0.10 \pm 0.02$ & $0.03 \pm 0.01$ & $-0.44 \pm 0.05$ \\
calculated & 0.06 & & 0.03 &
\end{tabular}

The low temperature coefficient of the GaAs modules means that, on warm days, its loss in performance compared to STC is smaller than for the $\mathrm{Si}$ module. An example time series of irradiance-corrected $P_{\mathrm{mp}}$, normalized to $P_{\mathrm{mp}, 0}$, is shown in Fig. 5 p. Here, $P_{\mathrm{mp}, 0}$, is taken from a corrected cold clear day measurement for the Si modules and an indoor measurement for the GaAs module The plot shows that in warm weather, the GaAs module's low power temperature coefficient enables it to operate at higher relative efficiency for most of the day. At noon on the example day, the GaAs module's normalized $P_{\mathrm{mp}}$ is $96 \%$ and the Si module's is $87 \%$. 

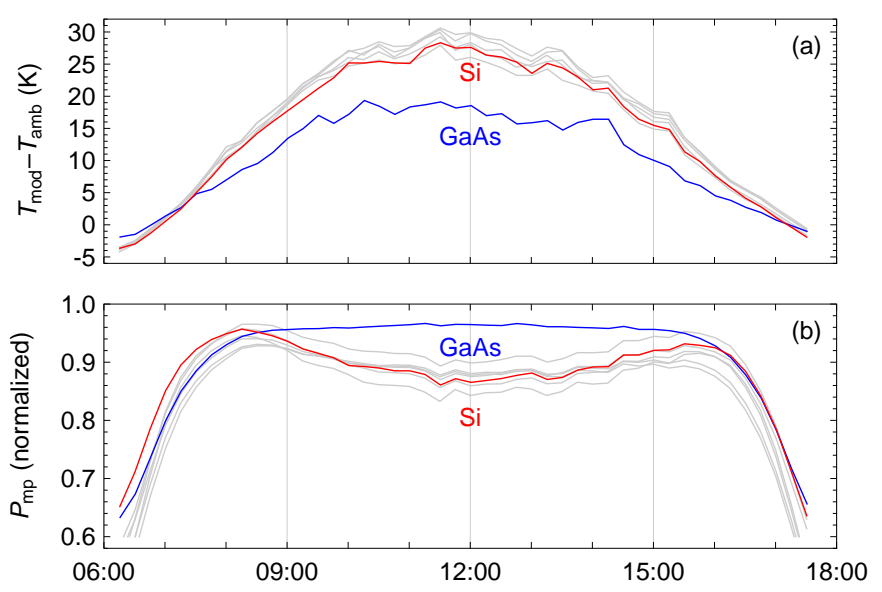

Fig. 5. (a) Module temperature rise above ambient and (b) irradiancecorrected $P_{\mathrm{mp}}$, normalized to $P_{\mathrm{mp}, 0}$, are plotted versus time. The light lines show data from five additional Si modules from various manufacturers. The data were collected on 9 September 2012, a completely clear-sky day with an average PWV of $7.8 \mathrm{~mm}$ and peak ambient temperature of $34^{\circ} \mathrm{C}$.

\section{B. Differences in operating temperature}

We observed that the GaAs module operated at a lower temperature than typical $\mathrm{Si}$ modules. The temperature rise above ambient is shown for a clear example day in Fig. 5a. Considering only high-irradiance conditions, the $\mathrm{Si}$ module deployed for the same time period as the GaAs module operated an average of $25.0^{\circ} \mathrm{C}$ above ambient temperature and the GaAs module operated an average of $18.4^{\circ} \mathrm{C}$ above ambient. $P_{\text {abs }}(E)$ and $P_{\text {excess }}(E)$, calculated according to Eqs. 1 and 2, are plotted for a Si module and the GaAs module in Fig. 6. For the Si module, the total power dissipated by nonphotocurrent-generating absorption is $307 \mathrm{~W} \mathrm{~m}^{-2}$, and the total power dissipated by excess photon energy of converted photons is $447 \mathrm{~W} \mathrm{~m} \mathrm{~m}^{-2}$. For the GaAs module, the total power dissipated by non-photocurrent-generating absorption is $340 \mathrm{~W} \mathrm{~m}^{-2}$, and the total power dissipated by excess photon energy of converted photons is $278 \mathrm{~W} \mathrm{~m}^{-2}$. Thus the total heating power dissipated in $\mathrm{Si}$ module is calculated to be $753 \mathrm{~W} \mathrm{~m}^{-2}$, and the total power dissipated as heat in the GaAs module is calculated to be $618 \mathrm{~W} \mathrm{~m}^{-2}$. Following the method described in [12], we calculated predicted operating temperatures of $25.5^{\circ} \mathrm{C}$ above ambient for the Si module and $20.1{ }^{\circ} \mathrm{C}$ above ambient for the GaAs module.

Based on these calculations, we attribute the reduced operating temperature of the GaAs module primarily to the reduction in heating from the excess energy of converted photons. This advantage is likely due both to the higher bandgap of GaAs (reducing thermalization loss) and the high quality of the absorber material, reducing non-radiative carrier recombination and enabling a high operating voltage. While the GaAs module has a higher reflectance than the Si module in the IR, its higher bandgap leads to an increase in the total sub-bandgap energy available to heat the module, and ultimately a higher overall total power dissipated by nonphotocurrent-generating absorption.

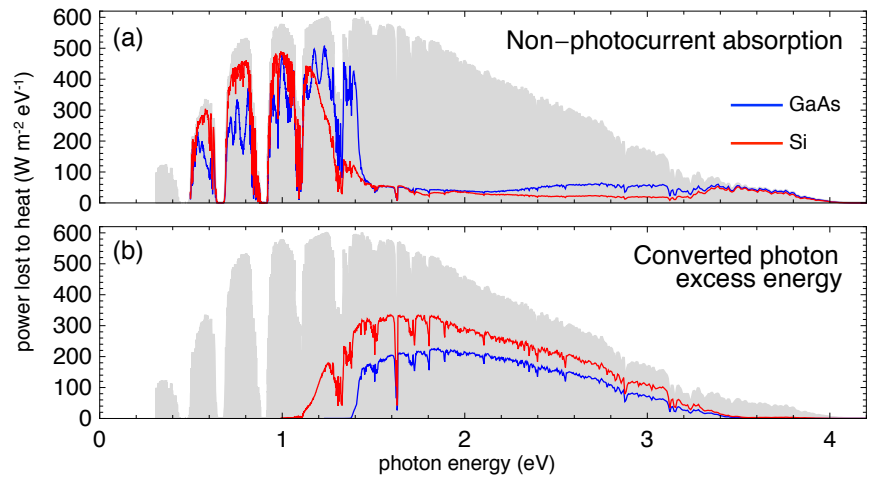

Fig. 6. Sources of heat dissipation in the GaAs and $\mathrm{Si}$ modules under maximum power operation calculated as functions of incident photon energy according to Eqs. 1 and 2 from indoor measurements of STC performance, EQE, and reflectance. (a) Heating from photons which are absorbed, but do not contribute to photocurrent $\left(P_{a b s}(E)\right)$. (b) Heating from the excess energy of photons which do contribute to photocurrent $\left(P_{\text {excess }}(E)\right)$

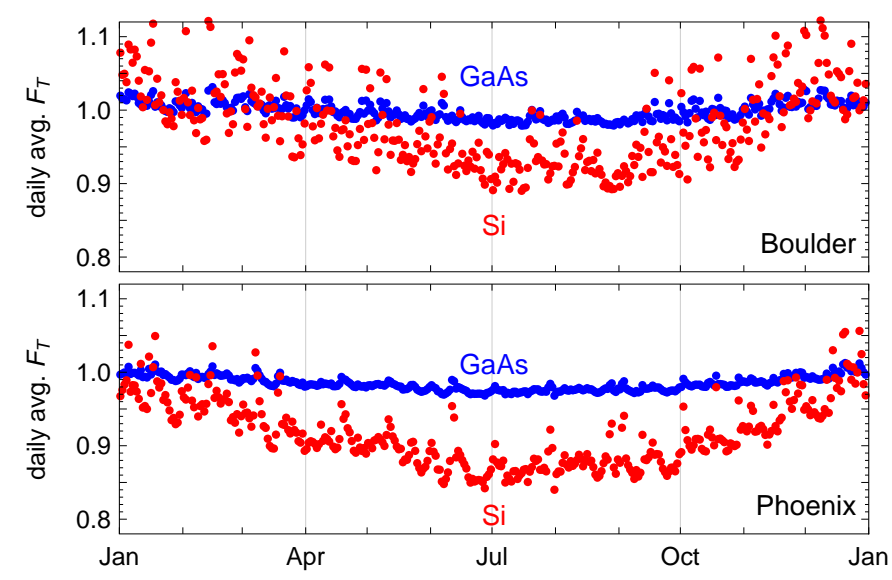

Fig. 7. Energy-weighted daily average temperature correction factor $F_{T}$ is plotted for TMY2 data in Boulder and Phoenix.

\section{Temperature effect on energy production}

$\bar{F}_{T}^{d}$, the energy-weighted daily average value for $F_{T}$, is plotted versus time in Fig. 7 The Si module shows a larger difference in performance between the warm and cold seasons in both locations and lower overall performance in Phoenix, where the ambient temperature is normally much higher. The effects of operating temperature and temperature coefficient only are thus expected to result in the GaAs module producing $(3 \pm 1) \%$ more energy per year in Boulder and $(8 \pm 2) \%$ more energy per year in Phoenix. This analysis does not consider the modules' different spectral responses or the different incident spectra in the two example locations.

\section{Irradiance effect on performance}

For the GaAs module, temperature- and irradiance-corrected power, normalized to power at STC, is plotted against irradiance in Fig. 8. The normalized power is $>90 \%$ of the one-sun value above $500 \mathrm{~W} / \mathrm{m}^{2}$. At lower irradiance, the data spread according to clearness index: low irradiance in a cloudy sky 
(low CI) produces better performance $(\geq 90 \%$ of the highirradiance performance) than low irradiance in a clear sky (high CI). This is because low irradiance in a clear sky occurs at sunrise and sunset, when the spectrum is red-rich.

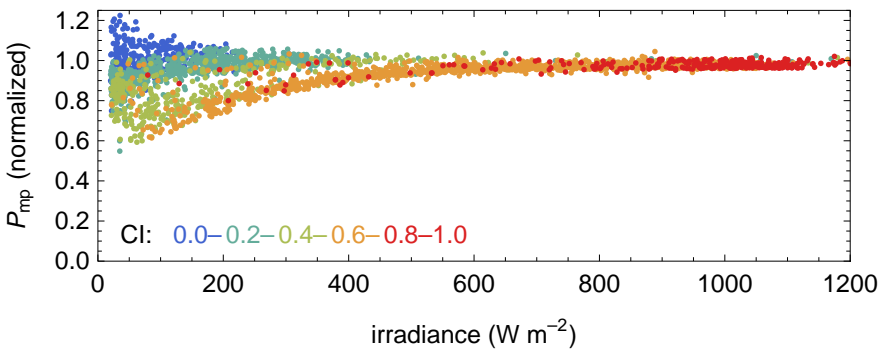

Fig. 8. $\quad P_{\mathrm{mp}}$ temperature- and irradiance-corrected and normalized to power at STC is plotted against irradiance. The color indicates the clearness index (CI) during each measurement.

\section{CONCLUSiON}

In the GaAs module, temperature-correlated spectral changes influence performance more strongly than temperature changes themselves. We showed that changes in spectrum and temperature are theoretically expected to affect different PV technologies to different degrees. This was borne out by analysis of outdoor measurements on a $\mathrm{Si}$ and a GaAs module.

Under our test conditions, for the GaAs module, irradiancecorrected power output is higher when module temperature is higher. We showed that this is due to the GaAs module's relatively small $V_{\mathrm{oc}}$ temperature coefficient and that temperaturecorrelated spectral effects dominate the $I_{\mathrm{sc}}$-temperature relationship. PWV was used to correct $I_{\mathrm{sc}}$ data for spectral changes and to derive spectrum-independent temperature coefficients that agree well with theoretical values and with a separate outdoor experiment. With the spectral correction, the $P_{\mathrm{mp}}$ temperature coefficient for the GaAs module is negative, but its magnitude is several times smaller than for the $\mathrm{Si}$ module. The reduced operating temperature and small $P_{\mathrm{mp}}$ temperature coefficient give the GaAs a performance advantage in warm weather or in adverse mounting conditions, such as in building-integrated applications. Considering only the differences in operating temperature and in temperature coefficient, compared to the $\mathrm{Si}$ module, the GaAs module is expected to produce annually $(3 \pm 1) \%$ more energy in Boulder and $(8 \pm 2) \%$ more energy in Phoenix when in an open-rack configuration. The performance of the GaAs module has a strong dependence on sky conditions at low irradiance, where for low values of $\mathrm{CI}$, performance is similar to the highirradiance performance.

The operating temperature of the GaAs module was substantially lower than that of the Si module because of a reduction in the heat generated by the excess energy of photocurrentgenerating photons. Further reductions in operating temperature may be achieved through improvements in infrared reflectivity.

\section{ACKNOWLEDGMENT}

This work was supported by the U.S. Department of Energy under Contract No. DE-AC36-08GO28308 with the National Renewable Energy Laboratory.

\section{REFERENCES}

[1] M. Green, K. Emery, Y. Hishikawa, W. Warta, and E. Dunlop, "Solar cell efficiency tables (version 41)," Progress in Photovoltaics: Research and Applications, vol. 21, no. 1, pp. 1-11, 2013.

[2] L. Mattos, S. Scully, M. Syfu, E. Olson, L. Yang, C. Ling, B. Kayes, and G. He, "New module efficiency record: $23.5 \%$ under 1-sun illumination using thin-film single-junction GaAs solar cells," in Photovoltaic Specialists Conference (PVSC), 2012 38th IEEE. IEEE, 2012, pp. 3187-3190.

[3] E. Yablonovitch, T. Gmitter, J. Harbison, and R. Bhat, "Extreme selectivity in the lift-off of epitaxial GaAs films," Applied Physics Letters, vol. 51, no. 26, pp. 2222-2224, 1987.

[4] B. Kayes, H. Nie, R. Twist, S. Spruytte, F. Reinhardt, I. Kizilyalli, and G. Higashi, " $27.6 \%$ conversion efficiency, a new record for singlejunction solar cells under 1 sun illumination," in Photovoltaic Specialists Conference (PVSC), 2011 37th IEEE. IEEE, 2011, pp. 4-8.

[5] ASTM Standard G173-03, Tables for Reference Solar Spectral Irradiances: Direct Normal and Hemispherical on 37 Tilted Surface. West Conshohocken, PA: ASTM International, 2012.

[6] M. Green, K. Emery, Y. Hishikawa, and W. Warta, "Solar cell efficiency tables (version 36)," Progress in Photovoltaics: Research and Applications, vol. 18, no. 5, pp. 346-352, 2010.

[7] ASTM Standard E 1036-08, Standard Test Methods for Electrical Performance of Nonconcentrator Terrestrial Photovoltaic Modules and Arrays Using Reference Cells. West Conshohocken, PA: ASTM International, 2008.

[8] Y. P. Varshni, "Temperature dependence of the energy gap in semiconductors," Physica, vol. 34, no. 1, pp. 149-154, 1967.

[9] C. D. Thurmond, "The standard thermodynamic functions for the formation of electrons and holes in $\mathrm{Ge}, \mathrm{Si}, \mathrm{GaAs}$, and $\mathrm{GaP}$,' Journal of the Electrochemical Society, vol. 122, no. 8, pp. 1133-1141, 1975.

[10] L. Nelson, M. Frichtl, and A. Panchula, "Changes in cadmium telluride photovoltaic system performance due to spectrum," Photovoltaics, IEEE Journal of, vol. 3, no. 1, pp. 488-493, 2013.

[11] A. Polman and H. A. Atwater, "Photonic design principles for ultrahighefficiency photovoltaics," Nat Mater, vol. 11, no. 3, pp. 174-177, Mar. 2012. [Online]. Available: http://dx.doi.org/10.1038/nmat3263

[12] M. Muller, B. Marion, and J. Rodriguez, "Evaluating the IEC 61215 Ed.3 NMOT procedure against the existing NOCT procedure with PV modules in a side-by-side configuration," in Photovoltaic Specialists Conference (PVSC), 2012 38th IEEE, 2012, pp. $000697-000702$. 